\title{
Facile Deoxygenation of Hydroxylated Flavonoids by Palladium-Catalysed Reduction of its Triflate Derivatives
}

\author{
József Kövér and Sándor Antus \\ Department of Organic Chemistry, University of Debrecen, H-4010 Debrecen, P. O. Box 20, Hungary \\ Reprint requests to Sándor Antus. Fax: +36-52-453-836. E-mail: antuss@tigris.klte.hu
}

Z. Naturforsch. 60b, 792 - 796 (2005); received November 12, 2004

Dedicated to Professor András Lipták on the occasion of his $70^{\text {th }}$ birthday

An efficient procedure to deoxygenate hydroxy substituted flavonoids, isoflavonoids and related compounds via their trifluoromethanesulfonates is presented. Their reduction with formic acid in the presence of a catalytic amount of palladium acetate, triethylamine and 1,3-bis(diphenylphosphanyl)propane (dppp) in DMF results in their des-hydroxy derivatives without affecting other functional groups.

Key words: Flavonoids, Reduction, Palladium(II) Acetate

Flavonoids and isoflavonoids possessing a 2- and 3-aryl substituted 1-benzopyran skeleton with various levels of saturation and oxidation, respectively, belong to the class of naturally occuring $O$-heterocyclic compounds [1] with wide range of biological importance due to their significant antioxidant [2], hepatoprotective [3], antifungal [4], antibacterical [5] and antiviral [6] activities.

Although, there are numerous independent methods available for the preparation of these natural products and the procedures of synthetic importance have been reviewed extensively $[1,7,8]$, these conditions are mostly suitable for direct synthesis of their 7hydroxy- or 5,7-dihydroxy derivatives and hence synthetic accessibility of their des-7-hydroxy derivatives is strongly limited. To overcome this problem several methods such as catalytic hydrogenation of $O$ aryl-N,N-dialkylisoureas by $\mathrm{Pd} / \mathrm{C}$ [9], desulfurization of S-aryl-N,N-dialkyl thiocarbamates (prepared by Newman-Kwart rearrangement of $O$-aryl-N,N-dialkyl thiocarbamates) by Raney nickel [10] and reduction of $O$-aryl (or methyl)- $p$-toluenesulfonates by $\mathrm{NaBH}_{4}-$ $\mathrm{NiCl}_{2}$ [11] or Raney nickel [12] have been recently introduced by Patonay et al. [13] in this field using 2,2dimethyl-7-hydroxychromanone ( 1a) as the substrate.

Efficient procedures for the deoxygenation of simple phenols have been reported via their trifluoromethanesulfonates (triflates) by hydrogenolysis under heterogeneous conditions ( $\mathrm{Pd} / \mathrm{C})$ [14] and $\mathrm{Ni}(0)-[15]$ or $\mathrm{Pd}(0)$-catalyzed $[16,17]$ reduction under homoge-

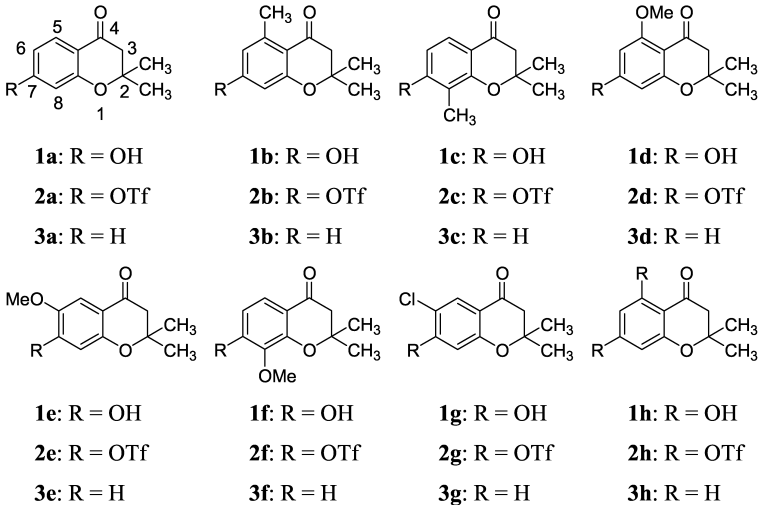

Scheme 1.

neous conditions. As a continuation of our research program aiming at the application of $\mathrm{Pd}(0)$-catalyzed transformation for the synthesis of $O$-heterocyclic compounds [18-20], it seemed promising to study also the deoxygenation of hydroxybenzopyranoid triflates by $\mathrm{Pd}(0)$-catalyzed reduction.

In order to compare our results with those published by Patonay et al. [13], 2,2-dimethyl-7-hydroxychromanone (1a) and its derivatives $\mathbf{1 b}-\mathbf{h}$ were chosen as model compounds (Scheme 1) whose corresponding triflates $\mathbf{2 a}-\mathbf{h}$ were prepared in high yields $(85-94 \%)$ by treatment with trifluoromethanesulfonic anhydride in the presence of triethylamine in dichloromethane at $-15^{\circ} \mathrm{C}$. When the reaction of 2,2dimethyl-chromanon-7-yl triflate (2a) with 1 equiv- 
Table 1. Compounds $\mathbf{3 a}-\mathbf{h}$ prepared.

\begin{tabular}{lcccccc}
\hline $\begin{array}{l}\text { Sub- } \\
\text { strate }\end{array}$ & Product & $\begin{array}{c}\text { Time } \\
{[\mathrm{min}]}\end{array}$ & $\begin{array}{c}\text { Yield }^{\mathrm{a}} \\
{[\%]}\end{array}$ & $\begin{array}{c}\text { M.p. } \\
{\left[{ }^{\circ} \mathrm{C}\right]}\end{array}$ & $\begin{array}{c}\text { Lit. m.p. } \\
{\left[{ }^{\circ} \mathrm{C}\right]}\end{array}$ & $\begin{array}{c}\text { Lit. yield }^{\mathrm{b}} \\
{[\%]}\end{array}$ \\
\hline $\mathbf{2 a}$ & $\mathbf{3 a}$ & 50 & 93 & $88.5-89$ & $89-90[13]$ & 80 \\
$\mathbf{2 b}$ & $\mathbf{3 b}$ & 25 & 90 & $68-69$ & $96.5[21]$ & 82 \\
$\mathbf{2 c}$ & $\mathbf{3 c}$ & 30 & 91 & oil & oil [22] & 80 \\
$\mathbf{2 d}$ & $\mathbf{3 d}$ & 70 & 95 & $124-125$ & $118-119[23]$ & 83 \\
$\mathbf{2 e}$ & $\mathbf{3 e}$ & 30 & 92 & $75-76$ & $71-72[23]$ & 80 \\
$\mathbf{2 f}$ & $\mathbf{3 f}$ & 20 & 91 & $132-133$ & $126-127[23]$ & 78 \\
$\mathbf{2 g}$ & $\mathbf{3 g}$ & 15 & 70.4 & oil & oil [24] & 41 \\
$\mathbf{2 h}$ & $\mathbf{3 h}$ & 30 & 75 & $88-89$ & $89-90[13]$ & 70 \\
\hline
\end{tabular}

a Isolated yields; ${ }^{\text {b }}$ ref. [13].

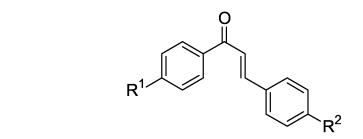

4a: $\mathrm{R}^{1}=\mathrm{OH}, \mathrm{R}^{2}=\mathrm{H} \quad$ 5a: $\mathrm{R}^{1}=\mathrm{H}, \mathrm{R}^{2}=\mathrm{OH}$

4b: $R^{1}=$ OTf, $R^{2}=H \quad 5 b: R^{1}=H, R^{2}=$ OTf

4c: $\mathrm{R}^{1}=\mathrm{R}^{2}=\mathrm{H}$
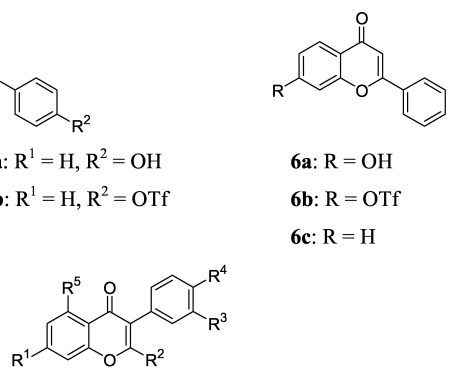

6a: $\mathrm{R}=\mathrm{OH}$

6b: $\mathrm{R}=\mathrm{OTf}$

6c: $\mathrm{R}=\mathrm{H}$

Table 2. Compounds $\mathbf{4 c - 1 1 c}$ prepared.

\begin{tabular}{cccccc}
\hline $\begin{array}{l}\text { Sub- } \\
\text { strate }\end{array}$ & Product & $\begin{array}{c}\text { Time } \\
{[\mathrm{min}]}\end{array}$ & $\begin{array}{c}\text { Yield }^{\mathrm{a}} \\
{[\%]}\end{array}$ & $\begin{array}{c}\text { M.p. } \\
{\left[{ }^{\circ} \mathrm{C}\right]}\end{array}$ & $\begin{array}{c}\text { Lit. m.p. } \\
{\left[{ }^{\circ} \mathrm{C}\right]}\end{array}$ \\
\hline $\mathbf{4 b}$ & $\mathbf{4 c}$ & 60 & 83 & $57-58$ & $60-62[25]$ \\
$\mathbf{5 b}$ & $\mathbf{5 c}$ & 90 & 83 & $56-57$ & $60-62[25]$ \\
$\mathbf{6 b}$ & $\mathbf{6 c}$ & 60 & 85 & $96-97$ & $97[26]$ \\
$\mathbf{7 b}$ & $\mathbf{7 c}$ & 30 & 90 & $134-135$ & $133-134[27]$ \\
$\mathbf{8 b}$ & $\mathbf{8 c}$ & 60 & 93 & $108-109$ & $86-87[28]$ \\
$\mathbf{9 b}$ & $\mathbf{9 c}$ & 60 & 90 & $150-151$ & $154-156[29]$ \\
$\mathbf{1 0 b}$ & $\mathbf{1 0 c}$ & 30 & 94 & $75-76$ & $75-78[30]$ \\
$\mathbf{1 1 b}$ & $\mathbf{1 1 c}$ & 90 & 79 & $66-67$ & $64-65[31]$ \\
\hline a Isolated yields. & & & &
\end{tabular}

but our procedure is significantly milder and simpler. Moreover, it has been also recognized that triflates 2a and $\mathbf{2 g}$ were less reactive towards the catalytic system using $\mathrm{PPh}_{3}$ instead of dppp. In both cases, hydrolysis of triflates $\mathbf{2 a}, \mathbf{g}$ to $\mathbf{1 a}, \mathbf{g}$, respectively, could also be observed besides their deoxygenation $(\mathbf{2 a} \rightarrow \mathbf{3 a}$, $\mathbf{2 g} \rightarrow \mathbf{3 g}$ ). In order to determine the scope and limitation of this deoxygenation procedure the transformation of the chalcones $\mathbf{4 b}$ and $\mathbf{5 b}$, the flavone $\mathbf{6 b}$, the isoflavones $\mathbf{7 b}-\mathbf{9 b}$, the coumarin $\mathbf{1 0 b}$ and chromone derivatives 11b was also studied (Scheme 2). The reduction of these compounds took place very smoothly to give the desired deoxygenated derivatives $4 \mathbf{c}-\mathbf{1 1 c}$, respectively, in good yields $(79-94 \%)$ and no byproducts could be detected (TLC) in any cases (Table 2).

In conclusion, these results have clearly indicated that the palladium(II) acetate/1,3-bis(diphenylphosphanyl)propane (dppp)/formic acid/triethylamine system in DMF is an efficient agent for deoxygenation of hydroxysubstituted flavonoids, isoflavonoids and related compounds via their trifluoromethanesulfonates.

\section{Experimental Section}

Melting points were determined on a Büchi 535 apparatus and are not corrected. ${ }^{1} \mathrm{H}$ NMR spectra were obtained on a Varian Gemini 200 NMR spectrometer in $\mathrm{CDCl}_{3}$ with TMS as internal standard. EI-MS $(70 \mathrm{eV})$ spectra were obtained with a VG TRIO-2 instrument. TLC was performed on Merck Kieselgel $60 \mathrm{~F}_{254}$ pre-coated aluminium plates. Elemental analyses $(\mathrm{C}, \mathrm{H})$ were conducted using Carlo Erba 1106 EA instrument. 7-Hydroxyflavone (6a) was purchased from Sigma-Aldrich. The preparation of the following derivatives was described earlier: 1a [22], 1b [32], 1c [22], 1d [13], 1e [33], 1f [13], 1g [34], 1h [35], 4a, 5a [36], 7a, 8a, 9a [37], 10a [38], 11a [39].

Aryl triflates. General procedure: 2,2-Dimethyl-7-(trifluoromethylsulfonyloxy)chromanone (2a)

To a solution of 7-hydroxy-2,2-dimethylchromanone [22] $(2.40 \mathrm{~g}, 12.50 \mathrm{mmol})$ in $20 \mathrm{ml}$ of dichloromethane and 
$3 \mathrm{ml}$ of triethylamine at $-15{ }^{\circ} \mathrm{C}$ was slowly added trifluoromethanesulfonic anhydride $(2.15 \mathrm{ml}, 3.70 \mathrm{~g}, 13.10 \mathrm{mmol})$. The mixture was stirred at this temperature for 5 minutes and then was allowed to warm to $23{ }^{\circ} \mathrm{C}$ and stirred until no starting material could be detected by TLC (30 minutes). The mixture was washed with conc. $\mathrm{NaHCO}_{3}$ solution $(2 \times 30 \mathrm{ml})$ and conc. $\mathrm{NaCl}$ solution, dried $\left(\mathrm{MgSO}_{4}\right)$, and concentrated. The residue was purified by chromatography [silica gel, elution with $n$-hexane : ethyl acetate (3:1)] to afford $2 \mathrm{a}$ as white crystals $(3.80 \mathrm{~g}, 94 \%)$; m. p. $29-30{ }^{\circ} \mathrm{C}$. ${ }^{1} \mathrm{H}$ NMR: $\delta=1.45$ (s, 6H, 2-H), 2.75 (s, 2H, 3-H), 6.90 (m, 2H, 6-H, 8-H), 7.95 (d, $J=9.28 \mathrm{~Hz}, 1 \mathrm{H}, 5-\mathrm{H}) .-\mathrm{MS}: m / e$ $(\%)=324\left(\mathrm{M}^{+}, 8\right), 309$ (22), 269 (12), 176 (19), 149 (19), 107 (39), 79 (32), 69 (100). $-\mathrm{C}_{12} \mathrm{H}_{11} \mathrm{~F}_{3} \mathrm{O}_{5} \mathrm{~S}$ (324.29): calcd. $\mathrm{C} 44.45, \mathrm{H} 3.42$; found C $44.40, \mathrm{H} 3.49$.

The compounds $\mathbf{2 b}-\mathbf{h}$ and $\mathbf{4 b} \mathbf{- 1 1} \mathbf{b}$ were prepared in an analogous manner.

\section{7-Trifluoromethylsulfonyloxy-2,2,5-trimethylchromanone} (2b)

(20 min, yield: $93 \%$, oil). $-{ }^{1} \mathrm{H}$ NMR: $\delta=1.45$ (s, $6 \mathrm{H}$, 2,2- $\left.\mathrm{CH}_{3}\right), 2.65\left(\mathrm{~s}, 3 \mathrm{H}, 5-\mathrm{CH}_{3}\right), 2.75(\mathrm{~s}, 2 \mathrm{H}, 3-\mathrm{H}), 6.65(\mathrm{~d}$, $J=2.80 \mathrm{~Hz}, 1 \mathrm{H}, 6-\mathrm{H}$ or $8-\mathrm{H}), 6.75(\mathrm{~d}, J=2.80 \mathrm{~Hz}, 1 \mathrm{H}, 6-\mathrm{H}$ or $8-\mathrm{H})$. - MS: $m / e(\%)=338\left(\mathrm{M}^{+}, 26\right), 323(10), 283(16)$, 121 (25), 93 (11), 69 (100). $-\mathrm{C}_{13} \mathrm{H}_{13} \mathrm{~F}_{3} \mathrm{O}_{5} \mathrm{~S}$ (338.30): calcd. C 46.15, H 3.87; found C 46.91, H 3.81.

\section{7-Trifluoromethylsulfonyloxy-2,2,8-trimethylchromanone} (2c)

(10 min, yield: $90 \%$, white crystals, m. p. $\left.53-54{ }^{\circ} \mathrm{C}\right)$. ${ }^{1} \mathrm{H}$ NMR: $\delta=1.50\left(\mathrm{~s}, 6 \mathrm{H}, 2,2-\mathrm{CH}_{3}\right), 2.25\left(\mathrm{~s}, 3 \mathrm{H}, 8-\mathrm{CH}_{3}\right)$, $2.75(\mathrm{~s}, 2 \mathrm{H}, 3-\mathrm{H}), 6.90(\mathrm{~d}, J=9.13 \mathrm{~Hz}, 1 \mathrm{H}, 5-\mathrm{H}$ or $6-\mathrm{H}), 7.80$ $(\mathrm{d}, J=9.10 \mathrm{~Hz}, 1 \mathrm{H}, 5-\mathrm{H}$ or $6-\mathrm{H}) .-\mathrm{MS}: m / e(\%)=338$ $\left(\mathrm{M}^{+}, 20\right), 323$ (51), 283 (22), 190 (25), 149 (29), 93 (11), 69 (100). $-\mathrm{C}_{13} \mathrm{H}_{13} \mathrm{~F}_{3} \mathrm{O}_{5} \mathrm{~S}$ (338.30): calcd. C 46.15, H 3.87; found $\mathrm{C} 46.31, \mathrm{H} 3.60$.

\section{2,2-Dimethyl-5-methoxy-7-(trifluoromethylsulfonyloxy)- chromanone (2d)}

(10 min, yield: $93 \%$, oil). $-{ }^{1} \mathrm{H}$ NMR: $\delta=1.45(\mathrm{~s}, 6 \mathrm{H}$, 2,2- $\left.\mathrm{CH}_{3}\right), 2.70(\mathrm{~s}, 2 \mathrm{H}, 3-\mathrm{H}), 3.90(\mathrm{~s}, 3 \mathrm{H}, 5-\mathrm{OMe}), 6.35$ (d, $J=2.17 \mathrm{~Hz}, 1 \mathrm{H}, 6-\mathrm{H}$ or $8-\mathrm{H}), 6.50(\mathrm{~d}, J=2.15 \mathrm{~Hz}, 1 \mathrm{H}, 6-\mathrm{H}$ or 8-H). - MS: $m / e(\%)=354\left(\mathrm{M}^{+}, 11\right), 339(10), 299(39)$, 206 (7), 138 (10), 83 (8), 69 (100). - $\mathrm{C}_{13} \mathrm{H}_{13} \mathrm{~F}_{3} \mathrm{O}_{6} \mathrm{~S}$ (354.30): calcd. C 44.07, H 3.69; found C 44.25, H 3.62.

\section{2,2-Dimethyl-6-methoxy-7-(trifluoromethylsulfonyloxy)- chromanone (2e)}

(10 min, yield: $89 \%$, white crystals, m.p. $\left.87-88{ }^{\circ} \mathrm{C}\right)$. ${ }^{1} \mathrm{H}$ NMR: $\delta=1.45$ (s, 6H, 2,2- $\left.\mathrm{CH}_{3}\right), 2.75$ (s, 2H, 3-H), 3.90 (s, 3H, 6-OMe), 6.90 (s, 1H, 8-H), 7.45 (s, 1H, 5-H). - MS: $m / e(\%)=354\left(\mathrm{M}^{+}, 8\right), 339(15), 299(8), 206(5), 165$ (6), 138 (32), 69 (100). $-\mathrm{C}_{13} \mathrm{H}_{13} \mathrm{~F}_{3} \mathrm{O}_{6} \mathrm{~S}$ (354.30): calcd. C 44.07, H 3.69; found C 44.50, H 3.59.

\section{2,2-Dimethyl-8-methoxy-7-(trifluoromethylsulfonyloxy)-} chromanone (2f)

(15 min, yield: $88 \%$, oil). $-{ }^{1} \mathrm{H}$ NMR: $\delta=1.55$ (s, $6 \mathrm{H}$, 2,2- $\mathrm{CH}_{3}$ ), 2.80 (s, 2H, 3-H), 4.05 (s, 3H, 8-OMe), 6.85 (d, $J=8.80 \mathrm{~Hz}, 1 \mathrm{H}, 6-\mathrm{H}), 7.65(\mathrm{~d}, J=8.80 \mathrm{~Hz}, 1 \mathrm{H}, 5-\mathrm{H}) .-\mathrm{MS}$ : $m / e(\%)=354\left(\mathrm{M}^{+}, 30\right), 339$ (41), 299 (31), 206 (25), 107 (100), 109 (28), 69 (100). - $\mathrm{C}_{13} \mathrm{H}_{13} \mathrm{~F}_{3} \mathrm{O}_{6} \mathrm{~S}$ (354.30): calcd. C 44.07, H 3.69; found C 44.32, H 3.70 .

6-Chloro-2,2-dimethyl-7-(trifluoromethylsulfonyloxy)chromanone $(\mathbf{2 g})$

(30 min, yield: $92 \%$, white crystals, m.p. $58-58.5^{\circ} \mathrm{C}$ ). ${ }^{1} \mathrm{H}$ NMR: $\delta=1.45$ (s, 6H, 2,2- $\left.\mathrm{CH}_{3}\right), 2.75(\mathrm{~s}, 2 \mathrm{H}, 3-\mathrm{H})$, $7.00(\mathrm{~s}, 1 \mathrm{H}, 8-\mathrm{H}), 8.00(\mathrm{~s}, 1 \mathrm{H}, 5-\mathrm{H}) .-\mathrm{MS}: m / e(\%)=358$ ( $\left.\mathrm{M}^{+}, 6\right), 343$ (12), 303 (6), 210 (10), 141 (12), 113 (9), 69 (100). $-\mathrm{C}_{12} \mathrm{H}_{10} \mathrm{ClF}_{3} \mathrm{O}_{5} \mathrm{~S}$ (358.72): calcd. C 40.17, H 2.80; found $\mathrm{C} 40.30, \mathrm{H} 2.95$.

\section{5,7-Bis(trifluoromethylsulfonyloxy)-2,2-dimethylchrom- anone $(\mathbf{2 h})$}

(30 min, yield: $85 \%$, white crystals, m. p. $123-124^{\circ} \mathrm{C}$ ). ${ }^{1} \mathrm{H}$ NMR: $\delta=1.51\left(\mathrm{~s}, 6 \mathrm{H}, 2,2-\mathrm{CH}_{3}\right), 2.80(\mathrm{~s}, 2 \mathrm{H}, 3-\mathrm{H})$, $6.73-6.74(\mathrm{~d}, J=2.20 \mathrm{~Hz}, 1 \mathrm{H}, 6-\mathrm{H}$ or $8-\mathrm{H}), 6.97-6.99$ (d, $J=2.29 \mathrm{~Hz}, 1 \mathrm{H}, 6-\mathrm{H}$ or $8-\mathrm{H}) .-\mathrm{C}_{13} \mathrm{H}_{10} \mathrm{~F}_{6} \mathrm{O}_{8} \mathrm{~S}_{2}$ (472.26): calcd. C 33.06, H 2.11; found C 33.21, H 2.16.

\section{4'-(Trifluoromethylsulfonyloxy)chalcone (4b)}

(20 min, yield: $94 \%$, white crystals, m. p. $96-97{ }^{\circ} \mathrm{C}$ ). ${ }^{1} \mathrm{H}$ NMR: $\delta=7.14-7.55(\mathrm{~m}, 6 \mathrm{H}$, aromatic- $\mathrm{H}, \alpha-\mathrm{CH})$, $7.60-7.75(\mathrm{~m}, 2 \mathrm{H}$, aromatic-H), 7.80-8.15 (m, 3H, aromatic-H, $\beta-\mathrm{CH}) .-\mathrm{MS}: m / e(\%)=356\left(\mathrm{M}^{+}, 42\right), 253$ (76), 131 (100), 69 (72). - $\mathrm{C}_{16} \mathrm{H}_{11} \mathrm{~F}_{3} \mathrm{O}_{4} \mathrm{~S}$ (356.32): calcd. C 53.93, H 3.11; found C 53.90, H 3.18.

\section{4-(Trifluoromethylsulfonyloxy)chalcone (5b)}

(15 min, yield: $95 \%$, white crystals, m.p. $60-61{ }^{\circ} \mathrm{C}$ ). ${ }^{1} \mathrm{H}$ NMR: $\delta=7.35$ (d, $J=8.72 \mathrm{~Hz}, 2 \mathrm{H}$, aromatic-H), $7.50(\mathrm{~d}, J=5.99 \mathrm{~Hz}, 2 \mathrm{H}$, aromatic-H), $7.56-7.66(\mathrm{~m}, 5 \mathrm{H}$, aromatic- $\mathrm{H}, \alpha$ - and $\beta-\mathrm{CH}), 8.03\left(\mathrm{dd}, J_{1}=1.37 \mathrm{~Hz}, J_{2}=\right.$ $8.71 \mathrm{~Hz}, 2 \mathrm{H}$, aromatic-H). - MS: $m / e(\%)=356\left(\mathrm{M}^{+}\right.$, 25), 223 (66), 131 (77), 105 (100), 69 (82). - $\mathrm{C}_{16} \mathrm{H}_{11} \mathrm{~F}_{3} \mathrm{O}_{4} \mathrm{~S}$ (356.32): calcd. C 53.93, H 3.11; found C 53.19, H 3.08.

\section{7-(Trifluoromethylsulfonyloxy)flavone (6b)}

(30 min, yield: $78 \%$, white crystals, m. p. $137-138{ }^{\circ} \mathrm{C}$ ). ${ }^{1} \mathrm{H}$ NMR: $\delta=6.87(\mathrm{~s}, 1 \mathrm{H}, 3-\mathrm{H}), 7.35\left(\mathrm{dd}, J_{1}=2.25 \mathrm{~Hz}, J_{2}=\right.$ 
$8.82 \mathrm{~Hz}, 1 \mathrm{H}, 6-\mathrm{H}), 7.51-7.59$ (m, 4H, aromatic-H), $7.91-$ $7.96(\mathrm{~m}, 2 \mathrm{H}$, aromatic-H), $8.35(\mathrm{~d}, J=8.80 \mathrm{~Hz}, 1 \mathrm{H}, 5-\mathrm{H}) .-$ MS: $m / e(\%)=370\left(\mathrm{M}^{+}, 80\right), 273(57), 268$ (54), 237 (100), 135 (55), 69 (75). $-\mathrm{C}_{16} \mathrm{H}_{9} \mathrm{~F}_{3} \mathrm{O}_{5} \mathrm{~S}$ (370.30): calcd. C 51.89, $\mathrm{H}$ 2.74; found C 51.01, H 2.79.

\section{7-(Trifluoromethylsulfonyloxy)isoflavone (7b)}

(45 min, yield: $89 \%$, white crystals, m. p. $146-147^{\circ} \mathrm{C}$ ). ${ }^{1} \mathrm{H}$ NMR: $\delta=7.26-7.57(\mathrm{~m}, 7 \mathrm{H}$, aromatic-H), $8.06(\mathrm{~s}$, $1 \mathrm{H}, 2-\mathrm{H}), 8.43(\mathrm{~d}, J=8.84 \mathrm{~Hz}, 1 \mathrm{H}, 5-\mathrm{H}) .-\mathrm{MS}: m / e$ $(\%)=370\left(\mathrm{M}^{+}, 52\right), 273(72), 268(88), 237$ (72), 135 (82), 69 (100). $-\mathrm{C}_{16} \mathrm{H}_{9} \mathrm{~F}_{3} \mathrm{O}_{5} \mathrm{~S}$ (370.30): calcd. C 51.89, H 2.74; found C 52.01, $\mathrm{H} 2.86$.

\section{5-Hydroxy-2-methyl-7-(trifluoromethylsulfonyloxy)- isoflavone $(\mathbf{8 b})$}

(30 min, yield: $68 \%$, white crystals, m. p. $128-129^{\circ} \mathrm{C}$ ). ${ }^{1} \mathrm{H}$ NMR: $\delta=2.30\left(\mathrm{~s}, 3 \mathrm{H}, \mathrm{CH}_{3}\right), 7.02(\mathrm{~s}, 1 \mathrm{H}, 8-\mathrm{H}), 7.35-$ $7.44(\mathrm{~m}, 6 \mathrm{H}$, aromatic-H), $13.14(\mathrm{~s}, 1 \mathrm{H}, 5-\mathrm{OH}) .-\mathrm{MS}: m / e$ $(\%)=400\left(\mathrm{M}^{+}, 32\right), 331$ (44), 303 (63), 284 (58), 267 (88), 151 (77), 69 (100). $-\mathrm{C}_{17} \mathrm{H}_{11} \mathrm{~F}_{3} \mathrm{O}_{6} \mathrm{~S}$ (400.33): calcd. C 51.00, $\mathrm{H} 2.76$; found C 51.17, H 2.85.

\section{3',4'-Methylenedioxy-7-(trifluoromethylsulfonyloxy)- isoflavone (9b)}

(30 min, yield: $73 \%$, white crystals, m. p. $174-175^{\circ} \mathrm{C}$ ). ${ }^{1} \mathrm{H}$ NMR: $\delta=6.01\left(\mathrm{~s}, 2 \mathrm{H},-\mathrm{OCH}_{2} \mathrm{O}-\right), 6.89(\mathrm{~d}, J=$ $8.01 \mathrm{~Hz}, 1 \mathrm{H}, 5$ '-H), $6.98\left(\mathrm{dd}, J_{1}=1.66 \mathrm{~Hz}, J_{2}=8.01 \mathrm{~Hz}\right.$, $\left.1 \mathrm{H}, 6^{\prime}-\mathrm{H}\right), 7.08$ (d, $\left.J=1.66 \mathrm{~Hz}, 1 \mathrm{H}, 2^{\prime}-\mathrm{H}\right), 7.35$ (dd, $J_{1}=$

[1] J. B. Harborn, T. J. Mabry, H. Mabry (eds): The Flavonoids, Chapman and Hall Ltd., London (1975).

[2] P. G. Pietta, J. Nat. Prod. 63, 1035 (2000).

[3] H. Wagner, in J.L. Bear, E. Reinhards (eds): Plant Constituents with Antihepatotoxic Activity in Natural Products as Medicinal Agents, p. 217, Hippokrates Verlag, Stuttgart (1980)

[4] J.A. M. Cruickshank, Aust. J. Biol. Chem. 15, 147 (1962).

[5] M. Hattori, S. Hada, A. Watahiki, H. Ihara, Y.Z. Shu, N. Kakiuchi, T. Mizuno, T. Namba, Chem. Pharm. Bull. 34, 3885 (1986).

[6] C. Q. Hu, K. Ke, Q. Shi, R.E. Kilkuskie, K. H. Lee, J. Nat. Prod. 57, 42 (1994).

[7] I. M. Lockhart, in G. P. Ellis (ed.): Chromenes, Chromanones and Chromones, p. 207, John Wiley and Sons Ltd., New York (1977).

[8] J. B. Harborn (ed.): The Flavonoids, Advances in Research since 1986, Chapman and Hall Ltd., London (1994).

[9] E. Vowinkel, H. J. Baese, Chem. Ber. 107, 1213 (1974). $\left.2.32 \mathrm{~Hz}, J_{2}=8.83 \mathrm{~Hz}, 1 \mathrm{H}, 6-\mathrm{H}\right), 7.46(\mathrm{~d}, J=2.32 \mathrm{~Hz}, 1 \mathrm{H}$, $8-\mathrm{H}), 8.02$ (s, 1H, 2-H), 8.42 (d, $J=8.83 \mathrm{~Hz}, 1 \mathrm{H}, 5-\mathrm{H})$. MS: $m / e(\%)=414\left(\mathrm{M}^{+}, 38\right), 281(88), 268$ (75), $252(72)$, 146 (51), 69 (100). $-\mathrm{C}_{17} \mathrm{H}_{9} \mathrm{~F}_{3} \mathrm{O}_{7} \mathrm{~S}$ (414.32): calcd. C 49.28, $\mathrm{H} 2.18$; found $\mathrm{C} 49.57 \mathrm{H} 2.23$.

\section{4-Methyl-7-(trifluoromethylsulfonyloxy)coumarin (10b)}

(30 min, yield: $91 \%$, white crystals, m.p. $83-84{ }^{\circ} \mathrm{C}$ ). ${ }^{1} \mathrm{H}$ NMR: $\delta=2.47\left(\mathrm{~d}, J=1.18 \mathrm{~Hz}, 3 \mathrm{H}, 4-\mathrm{CH}_{3}\right), 6.36-6.38$ (q, $J=1.18 \mathrm{~Hz}, 1 \mathrm{H}, 3-\mathrm{H}), 7.22(\mathrm{~d}, J=2.45 \mathrm{~Hz}, 1 \mathrm{H}, 8-\mathrm{H})$, $7.29\left(\mathrm{dd}, J_{1}=2.45 \mathrm{~Hz}, J_{2}=8.60 \mathrm{~Hz}, 1 \mathrm{H}, 6-\mathrm{H}\right), 7.70(\mathrm{~d}$, $J=8.60 \mathrm{~Hz}, 1 \mathrm{H}, 5-\mathrm{H}) .-\mathrm{MS}: m / e(\%)=308\left(\mathrm{M}^{+}, 46\right), 239$ (40), 175 (74), 69 (100). - $\mathrm{C}_{11} \mathrm{H}_{7} \mathrm{~F}_{3} \mathrm{O}_{5} \mathrm{~S}$ (308.23): calcd. C 42.86, H 2.29; found C 42.57, H 2.25.

Ethyl 7-(trifluoromethylsulfonyloxy)chromone-2-carboxylate (11b)

(30 min, yield: $66 \%$, white crystals, m.p. $127-128^{\circ} \mathrm{C}$ ). ${ }^{1} \mathrm{H}$ NMR: $\delta=1.45\left(\mathrm{t}, J=7.14 \mathrm{~Hz}, 3 \mathrm{H}\right.$, ethyl- $\left.\mathrm{CH}_{3}\right), 4.49$ (q, $J=7.14 \mathrm{~Hz}, 2 \mathrm{H}$, ethyl- $\left.\mathrm{CH}_{2}\right), 7.16(\mathrm{~s}, 1 \mathrm{H}, 3-\mathrm{H}), 7.37$ $\left(\mathrm{dd}, J_{1}=2.34 \mathrm{~Hz}, J_{2}=8.87 \mathrm{~Hz}, 1 \mathrm{H}, 6-\mathrm{H}\right), 7.65$ (d, $J=$ $2.34 \mathrm{~Hz}, 1 \mathrm{H}, 8-\mathrm{H}), 8.31(\mathrm{~d}, J=8.87 \mathrm{~Hz}, 1 \mathrm{H}, 5-\mathrm{H}) .-\mathrm{MS}$ : $m / e(\%)=336\left(\mathrm{M}^{+}, 20\right), 297$ (62), 268 (82), 233 (54), 69 (100). $-\mathrm{C}_{13} \mathrm{H}_{9} \mathrm{~F}_{3} \mathrm{O}_{7} \mathrm{~S}$ (366.27): calcd. C 42.63, H 2.47; found $\mathrm{C} 42.17, \mathrm{H} 2.50$.

\section{Acknowledgement}

We wish to thank the National Science Fundation (OTKA T049436) for financial support.

[10] H. Kwart, E. R. Evans, J. Org. Chem. 31, 410 (1966).

[11] F. Wang, K. Chiba, M. Tada, J. Chem. Soc. Perkin Trans. 1, 1897 (1992).

[12] G. W. Kenner, M. A. Murray, J. Chem. Soc. 178 (1949).

[13] P. Sebók, T. Tímár, T. Eszenyi, T. Patonay, J. Org. Chem. 59, 6318 (1994).

[14] A. G. Martinez, R. M. Alvarez, J. A. Aguirre, L.R. Subramarian, J. Chem. Soc. Perkin Trans 1, 1595 (1986).

[15] K. Sasaki, M. Sakai, Y. Sakakibara, K. Takagi, Chem. Lett. 2017 (1991).

[16] S. Cacchi, E. Morera, G. Ortar, Tetrahedron Lett. 25, 4821 (1986).

[17] S. Cacchi, E. Morera, G. Ortar, Org. Synth. 68, 138 (1990) and references therein.

[18] A. L. Tőkés, Gy. Litkei, K. Gulácsi, S. Antus, E. BaitzGács, Cs. Szántay, L. L. Darkó, Tetrahedron 55, 9283 (1999).

[19] L. Kiss, S. Antus, Heterocycl. Commun. 6, 309 (2000).

[20] L. Kiss, G. Papp, F. Joó, S. Antus, Heterocycl. Commun. 7, 417 (2001). 
[21] J. Cologne, R. Chambard, Bull. Soc. Chim. Fr. 20, 573 (1953).

[22] P. Sebők, J. Jekő, T. Tímár, J. Cs. Jászberényi, Heterocycles 38, 2099 (1994).

[23] F. Camps, J. Coll, O. Colomina, J. Heterocyclic. Chem. 22, 363 (1985).

[24] H. J. Kabbe, A. Widdig, Angew. Chem. 21, 254 (1982).

[25] R. D. Offenhauer, S. F. Nelsen, J. Org. Chem. 33, 775 (1968).

[26] A. Nishinaga, H. Ando, K. Maruyama, T. Mashino, Synthesis 839 (1992).

[27] A. Lévai, L. Balogh, Pharmazie 30, 747 (1975).

[28] A. Lévai, P. Sebők, Synth. Commun. 22, 1735 (1992).

[29] Gy. Litkei, R. Bognár, Z. Dinya, Chim. Acad. Sci. Hung. 71, 403 (1972).

[30] A. N. Brubaker, J. DeRuiter, W. L. Whitmer, J. Med. Chem. 29, 1094 (1986).
[31] G. P. Ellis, D. Shaw, J. Med. Chem. 15, 865 (1972).

[32] T. Tímár, J. Cs. Jászberényi, S. Hosztafi, Acta. Chim. Hung. 125, 457 (1988).

[33] F. Camps, J. Coll, A. Messguer, M. A. Pericas, S. Ricart, W.S. Bowers, D. M. Soderlund, Synthesis 725 (1980).

[34] P. Sebők, T. Tímár, J. Cs. Jászberényi, J. Jekő, Acta Chim. Hung. 126, 471 (1989).

[35] T. Tímár, J. Cs. Jászberényi, J. Heterocyclic Chem. 25, 871 (1988).

[36] H. Bablich, St. Kostanecki, Chem. Ber. 29, 233 (1897).

[37] A. Lévai, P. Sebők, Synth. Commun. 22, 1735 (1992).

[38] R. N. Lacey, J. Chem. Soc. 854 (1954).

[39] P. S. Manchand, R. A. Micheli, S. J. Saposnik, Tetrahedron 48, 9391 (1992). 